\title{
Maternal Criticism and Adolescent Depressive and Generalized Anxiety Disorder Symptoms: A 6-Year Longitudinal Community Study
}

\author{
Stefanie A. Nelemans • William W. Hale III • \\ Susan J. T. Branje • Skyler T. Hawk • Wim H. J. Meeus
}

Published online: 24 October 2013

(C) Springer Science+Business Media New York 2013

\begin{abstract}
This 6-year longitudinal study examined the direction of effects (i.e., parent effects, child effects, or reciprocal effects) between maternal criticism and adolescent depressive and Generalized Anxiety Disorder (GAD) symptoms, including adolescents' perceptions of criticism as a potential mediator. Consistent with recent empirical findings on associations between parenting and adolescent internalizing symptoms, we hypothesized stronger child effects than parent effects. A community sample of 497 adolescents ( $M_{\text {age }}=13.03$ at $\mathrm{T}_{1}, 57 \%$ boys) reported annually on their depressive and GAD symptoms as well as their perceptions of parental criticism. Their mothers $\left(M_{\text {age }}=\right.$ 44.41 at $\mathrm{T}_{1}$ ) also reported annually on their own critical behavior toward their adolescent. As expected, cross-lagged panel models demonstrated stronger child effects (i.e., adolescent psychopathology predicting maternal criticism) than parent effects (i.e., maternal criticism predicting adolescent psychopathology) for both adolescent depressive and GAD symptoms, including adolescent perceived criticism as a significant mediator. Our results emphasize the importance of considering (1) potential bidirectional influences over time, contrary to a focus on parent effects on adolescent mental health, as well as (2) adolescent perceptions of parenting as an important potential mediator in associations between aspects of the parent-adolescent relationship and adolescent internalizing psychopathological symptoms.
\end{abstract}

\footnotetext{
S. A. Nelemans $(\triangle) \cdot$ W. W. Hale III $\cdot$ S. J. T. Branje $\cdot$ S. T. Hawk W. H. J. Meeus

Research Centre Adolescent Development, Utrecht University, PO Box 80.140, 3508 TC Utrecht, The Netherlands e-mail: s.a.nelemans@uu.nl

W. H. J. Meeus

Department of Developmental Psychology, Tilburg University, PO Box 90.153, 5000 LE Tilburg, The Netherlands
}

Keywords Adolescence · Anxiety · Depression · Parental criticism (Expressed emotion) $\cdot$ Longitudinal $\cdot$ Cross-lagged panel model

Parental criticism has relatively recently become a topic of increased interest in research on parents' contributions to the development of adolescent psychopathology. Parental criticism refers to negative comments expressed by parents to their adolescent, and reflects a non-supportive emotional climate or interaction pattern within a specific parent-child dyad. More specifically, parental criticism is at the core of George Brown's theory of Expressed Emotion (or EE; Hooley and Teasdale 1989), further refined by Vaughn and Leff (1976, 1985). EE/criticism has received prominent attention in research on the development and maintenance of adult psychopathology, especially in relationship to schizophrenia and depression (Butzlaff and Hooley 1998). In contrast, the potential importance of parental EE/criticism in association with adolescent psychopathological symptoms has received relatively little attention in prior research, and this apparent gap in the literature is particularly true regarding adolescents from the general population. It persists despite theoretical expectations that parental EE may indeed be an important factor in the (further) development of adolescent psychopathology, because of the continued importance of the parent-child relationship for adolescent adjustment (Laursen and Collins 2009) and the fact that adolescents' psychopathological symptoms typically first occur when they are living with their parents (Asarnow et al. 1993; Hale et al. 2011b).

Symptoms of anxiety and depression are especially important to consider in the association between parental criticism and adolescent psychopathology, because these are among the most prevalent forms of psychopathology during adolescence (Merikangas et al. 2010). These internalizing problems have also shown strong links to problematic 
parent-adolescent interactions (Hughes and Gullone 2008), of which parental criticism is indicative (McCarty et al. 2004). The existing research thus far has focused mainly on parental criticism as a predictor of adolescent symptoms of anxiety and depression, acting on the presumption that high parental criticism may contribute to the onset of, or increases in, these forms of adolescent psychopathology. These studies have demonstrated that high parental criticism appears to be related to concurrent and future symptoms of anxiety and depression in children and adolescents (e.g., Asarnow et al. 1993, 2001; Frye and Garber 2005; Gar and Hudson 2008; Hale et al. 2011c; Schwartz et al. 1990; Silk et al. 2009; Tompson et al. 2010). By solely focusing on contributions of parental criticism to adolescent symptoms of anxiety and depression (i.e., a parent effects model; Branje et al. 2008), however, studies have largely ignored potentially important adolescent characteristics that may evoke, reinforce, and/or shape parental criticism (i.e., a child effects model; Branje et al. 2008; Kerr and Stattin 2003), as well as potential bidirectional influences between parental criticism and adolescent psychopathological symptoms (i.e., a reciprocal effects model; Branje et al. 2008; Lollis and Kuczynski 1997). Child effects and reciprocal effects may be especially important to consider during adolescence, when there is a realignment of the parent-child relationship. This process is characterized by adolescent separation, individuation, and increasing autonomy and as a result of this process, the parent-adolescent relationship becomes more egalitarian and reciprocal (De Goede et al. 2009).

Systematic support for child effects or reciprocal effects models comes from recent studies on other aspects of parenting in association with adolescent anxiety and depressive symptoms (e.g., Branje et al. 2010; Van Eijck et al. 2012; Wijsbroek et al. 2011). All of these aforementioned studies have demonstrated that adolescent psychopathological symptoms were significantly predictive of a more negative parent-adolescent relationship later on, suggesting that adolescent characteristics may indeed evoke, reinforce, and/or shape negative interaction patterns between adolescents and their parents. Prospective longitudinal studies of potential bidirectional associations between the specific construct of parental criticism and adolescent anxiety and depressive symptoms, which are needed to answer questions of direction of effects, are however scarce (Frye and Garber 2005; Hale et al. 2011b). Examining the direction of effects between parental criticism and adolescent symptoms of anxiety and depression is not only of theoretical interest, but also of practical importance, as evidence of specific effects (either parent-driven, child-driven, or bidirectional) may guide prevention and interventions aimed at ameliorating both parental criticism and adolescent anxiety/depressive symptoms. Therefore, in this study we aimed to examine the direction of effects between parental criticism and adolescents' anxiety/depressive symptoms in a prospective longitudinal community study covering the period from early to late adolescence.

As most previous EE studies have been either crosssectional or conducted in designs in which parental criticism at one point in time predicted later adolescent psychopathological symptoms, these studies appear to presume that parental criticism is a manifestation of a parental trait rather than a construct involving change over time. The developmental properties of the EE construct itself have therefore largely been ignored (Hale et al. 2011c). Even though parenting may be fairly stable over time (e.g., indicated by relatively high rank-order stability; Holden and Miller 1999), this does not imply that there are no potential dynamic changes over time. Parents will naturally respond to the actions of their children (Branje et al. 2008; Kerr and Stattin 2003). This is also supported by studies demonstrating changes in parental EE after child therapy (Gar and Hudson 2009), and the potential for adolescents' characteristics to evoke, reinforce, and/or shape parenting behaviors (e.g., Branje et al. 2010; Van Eijck et al. 2012; Wijsbroek et al. 2011). Repeated measurements of parental criticism over a period of time may thus not only shed light on whether dynamic changes in parental behaviors and attitudes predict adolescent anxiety and depression (i.e., parent effects), but also on whether changes in adolescent anxiety and depression predict later parental criticism (i.e., child or reciprocal effects).

Another largely ignored issue regarding parental criticism concerns the importance of adolescents' perceptions of parental EE. Adolescents' subjective experience of criticism may have just as much, or perhaps even more, influence on their mental health or the parent-adolescent relationship as the amount of criticism they receive according to their parents (as discussed in Hale et al. 2007). The importance of children's perceptions of parental rejection in association with children's psychological adjustment is also stressed in Rohner's Parental Acceptance-Rejection Theory (Rohner et al. 2005). Additionally, a study by Bolton et al. (2009) has further emphasized the significance of adolescents' perceptions of parental criticism in understanding the relationship between parental criticism and adolescents' depressive symptoms. Until now, however, relatively little research on adolescent psychopathology has addressed the interrelationship between parent-reported critical behavior, which is far from a perfect predictor of adolescents' psychopathological symptoms, and adolescents' own perceptions of parental criticism.

A likely mechanism relating parental criticism and adolescent psychopathological symptoms may be adolescents' perception of received parental criticism. Many studies have argued that adolescent perceptions of (negative) parenting may be a critical mediating variable in parent effects models examining associations between parenting and adolescent psychopathological symptoms (Millikan et al. 2002; Powers 
et al. 1994). In addition, studies have suggested mediation in child effects models by showing that adolescent psychopathological symptoms may negatively affect perceptions of parenting (e.g., Yahav 2007), which may (further) deteriorate the emotional climate between parents and adolescents. Perhaps adolescents' emotional problems elicit negative emotional reactions from parents, partially via adolescents' negative appreciations of the parent-child relationship or emotional climate (which may or may not mirror objectively more difficult interactions with their parents). Altogether, these studies suggest the potential importance of adolescents' perceptions in the associations between parental criticism and youths' anxiety and depressive symptoms. In the present study, we therefore examined whether adolescent perceptions of parental criticism mediated the potential bidirectional associations between mother-reported criticism and adolescents' anxiety and depressive symptoms over time.

Even though there is overwhelming evidence that anxiety and depression are strongly related (Brady and Kendall 1992), which seems to be especially true for symptoms of depression and Generalized Anxiety Disorder (GAD), other studies have argued that anxiety and depression also represent distinct entities (e.g., Hale et al. 2009). Despite the substantial overlap between depression and GAD, these symptoms may be associated with different parent-adolescent interaction processes. Two meta-analytic overviews by McLeod and colleagues $(2007 \mathrm{a}, \mathrm{b})$, for example, seem to suggest that parental rejection/aversiveness (including criticism) may be more important in association with child and adolescent depressive symptoms than anxiety symptoms, but it remains unclear whether parental criticism is differentially associated with adolescent symptoms of depression and GAD over time. Therefore, this study specifically focused on the potential bidirectional associations between maternal criticism and adolescent depressive and GAD symptoms over time.

Furthermore, most previous EE studies have been conducted within clinical settings, while much less attention has been given to the associations between parental EE and anxiety and depressive symptoms in adolescents from the general population. Studies with adolescent samples from the general population are important, because a potential referral bias limits the generalizability of research findings in clinical samples (e.g., Hale et al. 2005). In addition, findings in the general population may provide important insights into mechanisms potentially affecting adolescent psychopathological symptoms before their severity has reached an actual diagnosis, which may then become target for prevention and intervention.

\section{Present Study}

In the present 6-year longitudinal community study, we aimed to examine the direction of effects (i.e., parent effects, child effects, or reciprocal effects) between maternal criticism and adolescent depressive and GAD symptoms, including adolescents' perceptions of criticism as a potential mediator in the associations. In a parent effects model, we would expect maternal criticism to positively predict adolescents' depressive and GAD symptoms over time. In contrast, in a child effects model we would expect adolescents' depressive and GAD symptoms to positively predict maternal criticism over time. In a reciprocal effects model, we would expect to find both of the aforementioned parent and child effects over time.

Secondly, we hypothesized that the longitudinal association between maternal criticism and adolescent depression/GAD would be mediated by adolescents' perceptions of criticism. In a parent effects model, mediation of adolescent perceived EE in the longitudinal association from maternal EE to adolescent depression/GAD would suggest that maternal criticism negatively affects adolescents' perceptions of the emotional climate or mother-adolescent interaction patterns, which in turn affect adolescents' depressive and GAD symptoms over time. In contrast, mediation of adolescent perceived EE in a child effects model would suggest that adolescents' depressive and GAD symptoms affect their perceptions of the emotional climate or mother-adolescent interaction patterns, which in turn affect maternal criticism. In a reciprocal effects model, both of the aforementioned processes may be taking place.

Finally, based on recent studies that have found stronger support for child effects than parent effects in associations between different aspects of parenting and adolescent anxiety and depressive symptoms (e.g., Branje et al. 2010; Van Eijck et al. 2012; Wijsbroek et al. 2011), we also hypothesized stronger longitudinal child effects from adolescents' depression and GAD to maternal criticism, compared to the longitudinal parent effects from maternal criticism to adolescents' depression and GAD. This study adds to the existing literature by addressing the limited attention in previous EE/criticism studies to longitudinal and potentially bidirectional associations between parental criticism reported by both mothers and adolescents and adolescent depression/ GAD symptoms in the general population.

\section{Method}

Participants

Participants in this 6-year longitudinal study were 497 adolescents (57\% boys), with a mean age of 13.03 years $(S D=0.46)$ who attended the first year of secondary school at the start of the study, and their mothers, with a mean age of 44.41 years $(S D=4.45$, ranging from 31 to 64 years) at the start of the study. All adolescents identified themselves as being ethnic Dutch. Data for this study were part of the larger ongoing 
Research on Adolescent Development And Relationships (RADAR) project.

Sample attrition was low across waves, with 425 of the 497 adolescents (attrition of approximately $3.1 \%$ every wave) and 419 of the 497 mothers (attrition of approximately $3.3 \%$ every wave) still participating at the sixth annual measurement wave. Adolescents participating at all six measurement waves were slightly younger than those dropping out of the study, $F(1,495)=5.45, p=0.02, \eta^{2}=0.01$, but there were no significant differences in gender, $\chi^{2}(1)=0.60, p=0.44$, Cramer's $V=0.04$, or in other variables of interest at the start of the study, $F(4,464)=1.93, p=0.10, \eta^{2}=0.02$. Little's Missing Completely at Random (MCAR) test indicated a good fit between sample scores with and without imputation, $\chi^{2}(1053)=1073.53, p=0.32$. Incidental missing item values were estimated in SPSS, using Expectation Maximization (EM). Remaining missing data were handled in Mplus using the Full Information Maximum Likelihood procedure (FIML; Muthén and Muthén 2010).

\section{Procedure}

The participants were recruited from randomly selected schools in the western and central regions of the Netherlands. Before the start of the study, adolescents and their parents received a complete description of the study and provided active written informed consent to participate. For six successive years, adolescents and their mothers completed annual self-report questionnaires during a home visit. Participants received a small monetary compensation for every wave they completed the questionnaires. This study was approved by the Board of the local research institute and by the Medical Ethical Committee of the Utrecht Medical Centre.

\section{Measures}

Depressive Symptoms We used the Dutch version of Reynolds Adolescent Depression Scale, second edition (RADS-2; Reynolds 2000) to assess adolescent depressive symptoms. The RADS-2 is a self-report questionnaire that consists of 23 items, which are measured on a 4-point scale ranging from 1 (almost never) to 4 (usually). Sample items include "I am sad" and "I feel like crying". In this study, internal consistency for this scale was found to be good across waves, with Cronbach's alpha ranging between 0.93 and 0.95 . Previous studies have shown good psychometric properties for the RADS-2 among adolescents (Osman et al. 2010).

GAD Symptoms We used the Dutch version of the original 38item Screen for Child Anxiety Related Emotional Disorders (SCARED; Birmaher et al. 1997; Hale et al. 2005) to assess adolescent GAD symptoms. The SCARED is a self-report questionnaire measuring four anxiety disorder symptom dimensions in children and adolescents that are directly related to the individual anxiety disorders in the DSM-IV-TR (American Psychiatric Association 2000), including a 9-item subscale measuring symptoms of GAD. Items were rated on a 3-point scale, ranging from 1 (almost never) to 3 (often). Sample items include "I worry about how well I do things" and "I worry about the future". In this study, internal consistency for the GAD subscale was found to be good across waves, with Cronbach's alpha ranging between 0.85 and 0.91 . Psychometric properties of the SCARED have shown to be good in several previous studies (Birmaher et al. 1997; Hale et al. 2005; for a meta-analysis on the psychometric properties of the SCARED, see Hale et al. 2011a).

Parental Criticism We used the Dutch version of both the parent and adolescent version of the 38-item Level of Expressed Emotions Scale (LEE; Gerlsma and Hale 1997; Hale et al. 2007) to assess parental criticism as reported by mothers and as perceived by adolescents. The LEE is a selfreport questionnaire with a 5-item subscale measuring the $\mathrm{EE}$ dimension of criticism. Items were rated on a 4-point scale, ranging from 1 (untrue) to 4 (true). Sample items include "My parents are critical of me/I am critical of my child" and "My parents try to change me/I try to change my child". In this study, Cronbach's alpha internal consistency ranged between 0.73 and 0.81 across waves for adolescent reports, and from 0.57 to 0.59 across waves for mother reports. Psychometric properties of the 38-item LEE (mainly using the patient/ adolescent version, not the relative/parent version) have shown to be good in several previous studies (Gerlsma and Hale 1997; Hale et al. 2007).

\section{Results}

\section{Descriptive Statistics}

Table 1 provides an overview of mean levels of all study variables over the studied period of 6 years. Rank-order stability between successive waves was high for all variables, with correlations ranging between 0.56 and 0.67 for maternal criticism, ranging between 0.56 and 0.68 for adolescent perceived criticism, ranging between 0.57 and 0.76 for depressive symptoms, and ranging between 0.59 and 0.76 for GAD symptoms. Furthermore, within-wave correlations between adolescent perceived criticism and depressive symptoms (ranging between 0.35 and 0.45 ) and GAD symptoms (ranging between 0.15 and 0.24 ) appeared to be somewhat stronger than within-wave correlations between maternal criticism and depressive symptoms (ranging between 0.16 and 0.24 ) and GAD symptoms (ranging between 0.01 and 0.15 ). Finally, within-wave correlations between 
Table 1 Mean levels and standard deviations of all study variables over six annual measurement waves

\begin{tabular}{|c|c|c|c|c|c|c|c|c|c|c|c|c|}
\hline \multirow[t]{2}{*}{ Variable } & \multicolumn{2}{|c|}{ Time 1} & \multicolumn{2}{|c|}{ Time 2} & \multicolumn{2}{|c|}{ Time 3} & \multicolumn{2}{|c|}{ Time 4} & \multicolumn{2}{|c|}{ Time 5} & \multicolumn{2}{|c|}{ Time 6} \\
\hline & $M$ & $S D$ & $M$ & $S D$ & $M$ & $S D$ & $M$ & $S D$ & $M$ & $S D$ & $M$ & $S D$ \\
\hline Maternal criticism & 1.58 & 0.40 & 1.56 & 0.39 & 1.54 & 0.38 & 1.54 & 0.39 & 1.55 & 0.40 & 1.53 & 0.39 \\
\hline Adolescent perceived criticism & 1.65 & 0.50 & 1.72 & 0.54 & 1.78 & 0.56 & 1.81 & 0.56 & 1.79 & 0.59 & 1.77 & 0.57 \\
\hline Depression & 1.63 & 0.49 & 1.51 & 0.50 & 1.54 & 0.52 & 1.56 & 0.54 & 1.55 & 0.51 & 1.59 & 0.55 \\
\hline GAD & 1.38 & 0.39 & 1.33 & 0.38 & 1.36 & 0.43 & 1.39 & 0.44 & 1.38 & 0.44 & 1.42 & 0.47 \\
\hline
\end{tabular}

GAD Generalized Anxiety Disorder. Maternal criticism and adolescent perceived criticism were measured on a scale from 1 to 4 . Depressive symptoms were measured on a scale from 1 to 4 . GAD symptoms were measured on a scale from 1 to 3

adolescent perceived criticism and maternal criticism were moderate (ranging between 0.28 and 0.39 ), while withinwave correlations between adolescent depressive and GAD symptoms were high (ranging between 0.69 and 0.78$){ }^{1}$

\section{Statistical Analysis}

To address our research questions, we used structural equation modeling (SEM) in Mplus version 7.11 (Muthén and Muthén 2010) using six waves of longitudinal data on maternal criticism, adolescent perceived criticism, and - in separate models - adolescent depressive symptoms and adolescent GAD symptoms. Maximum likelihood estimation with standard errors and chi square robust to non-normality was used (MLR estimator). In addition, we used the analytical strategy for comparing differences between indirect effects described in Preacher and Hayes (2008) to test whether the indirect parent effects and child effects were significantly different from each other.

Two separate cross-lagged panel models were estimated for adolescent depressive symptoms and adolescent GAD symptoms, respectively, in order to examine longitudinal bidirectional associations between maternal criticism and these symptoms, including adolescent perceived criticism as a mediator in the associations. In these models, we included sex as a covariate by regressing all variables on the covariate representing sex.

We followed a stepwise method to construct the best fitting cross-lagged panel model for adolescent depressive symptoms and adolescent GAD symptoms. We first began our analyses with a model that included the 1 year and 2 year stability paths for all variables, and all $\mathrm{T}_{1}$ correlations between the variables. Next, we added all concurrent (within-time) correlations between the variables over time, which significantly improved the model fit for both depression, $\Delta \chi_{\mathrm{SB}}^{2}(12)=221.88$, $p<0.001$, and GAD, $\Delta \chi_{\mathrm{SB}}^{2}(12)=102.77, p<0.001$. Then, we separately added longitudinal cross-lagged parent effects from maternal criticism to adolescent perceived criticism

\footnotetext{
${ }^{1}$ The full correlation matrix is available from the first author on request.
}

1 year later, which significantly improved the model fit for both depression, $\Delta \chi^{2}{ }_{\mathrm{SB}}(5)=19.46, p=0.002$, and GAD, $\Delta \chi_{\mathrm{SB}}^{2}(5)=20.02, p=0.001$, and longitudinal cross-lagged parent effects from adolescent perceived criticism to adolescent symptoms of depression or GAD 1 year later, which significantly improved the model fit for depression, $\Delta \chi_{\mathrm{SB}}^{2}(5)=18.22, p=0.003$, but not for GAD, $\Delta \chi_{\mathrm{SB}}^{2}(5)=$ $5.58, p=0.35$. We then separately added longitudinal crosslagged child effects from adolescent symptoms of depression or GAD to adolescent perceived criticism 1 year later, which significantly improved the model fit for both depression, $\Delta \chi_{\mathrm{SB}}{ }_{\mathrm{SB}}(5)=56.11, p<0.001$, and GAD, $\Delta \chi^{2}{ }_{\mathrm{SB}}(5)=37.87$, $p<0.001$, and longitudinal cross-lagged child effects from adolescent perceived criticism to maternal criticism 1 year later, which significantly improved the model fit for both depression, $\Delta \chi^{2}{ }_{\mathrm{SB}}(5)=37.40, p<0.001$, and GAD, $\Delta \chi_{\mathrm{SB}}^{2}(5)=41.44, p<0.001$. Finally, we separately added longitudinal direct parent effects from maternal criticism to adolescent symptoms of depression or GAD 1 year later, which did not significantly improve the model fit for either depression, $\Delta \chi_{\mathrm{SB}}^{2}(5)=2.98, p=0.70$, or GAD, $\Delta \chi_{\mathrm{SB}}^{2}(5)=$ $6.28, p=0.28$, and longitudinal direct child effects from adolescent depression or GAD to maternal criticism 1 year later, which did not significantly improve the model fit for either depression, $\Delta \chi_{\mathrm{SB}}^{2}(5)=2.93, p=0.71$, or $\mathrm{GAD}$, $\Delta \chi_{\mathrm{SB}}^{2}(5)=1.46, p=0.92$.

Including all of the above described significant paths resulted in models with good fit for both adolescent depressive symptoms, $\chi_{\mathrm{SB}}^{2}(88)=155.79, \mathrm{CFI}=0.98, \mathrm{RMSEA}=0.04$, $90 \%$ CI of RMSEA [0.029, 0.049], and adolescent GAD symptoms, $\chi_{\mathrm{SB}}^{2}(93)=175.99, \mathrm{CFI}=0.98, \mathrm{RMSEA}=0.04$, $90 \%$ CI of RMSEA [0.033, 0.052]. Subsequently, we tested whether all the paths (i.e., 1 year and 2 year stability effects, within-time correlations, cross-lagged effects, and regressions of all variables on the covariate representing sex) were time-invariant, by constraining the same paths to be equal to each other over time. Constraining all paths to be equal over time did not significantly worsen the model fit for either depression, $\Delta \chi_{\mathrm{SB}}^{2}(64)=78.47, p=0.11$, or GAD, $\Delta \chi_{\mathrm{SB}}^{2}(60)=68.15, p=0.22$. This modification was therefore 
retained and resulted in the final cross-lagged panel model with good fit for both adolescent depressive symptoms, $\chi_{\mathrm{SB}}^{2}(152)=233.69, \mathrm{CFI}=0.98, \mathrm{RMSEA}=0.03,90 \% \mathrm{CI}$ of RMSEA [0.024, 0.041], and adolescent GAD symptoms, $\chi_{\mathrm{SB}}^{2}(153)=243.16, \mathrm{CFI}=0.98, \mathrm{RMSEA}=0.03,90 \% \mathrm{CI}$ of RMSEA [0.026, 0.042]. In addition, in these models large proportions of variance were explained in all variables (i.e., $33.5 \%-59.7 \%$ in depressive symptoms, $30.1 \%-48.9 \%$ in adolescent perceived criticism in the depression model, and $26.6 \%-53.0 \%$ in maternal criticism in the depression model; $35.4 \%-61.0 \%$ in GAD symptoms, $28.8 \%-47.6 \%$ in adolescent perceived criticism in the GAD model, and $26.6 \%-53.1 \%$ in maternal criticism in the GAD model).

In these final models, the adolescent's sex appeared to significantly predict depressive symptoms ( $\beta$ s ranging between 0.10 and 0.11 ) and GAD symptoms ( $\beta$ s ranging between 0.09 and 0.11$)$, as well as the adolescent's perceived criticism $(\beta \mathrm{s}$ ranging between -0.07 and -0.08 in both the GAD model and the depression model). These effects indicated that girls generally reported more depressive symptoms, more GAD symptoms, and less perceived criticism, compared to boys. Using multigroup analyses, we also explored potential moderation by sex of the longitudinal associations in the final models. Results suggested no significant moderation of the cross-lagged paths, as freeing these paths between boys and girls did not significantly improve the model fit for either depressive symptoms, $\Delta \chi_{\mathrm{SB}}^{2}(4)=2.60, p=0.63$, or GAD symptoms, $\Delta \chi_{\mathrm{SB}}^{2}(3)=3.19, p=0.36$.

In the final models controlling for sex, we tested for potential longitudinal indirect effects from maternal criticism to adolescent depressive or GAD symptoms, via adolescent perceived criticism, and the reverse longitudinal indirect effects from adolescent depressive or GAD symptoms to maternal criticism, via adolescent perceived criticism. All estimations of the longitudinal indirect effects were based on the Mplus estimation of indirect effects (Muthén and Muthén 2010). Since MLR estimation corrects standard errors of estimates in a comparable manner as bootstrapping does, bootstrapped effects are not available in Mplus in combination with MLR estimation because MLR estimates are comparable to bootstrapped estimates with regular ML estimation (Muthén and Muthén 2010). Nonetheless, we checked our final MLR estimation models with ML estimation models in combination with 10,000 bootstraps, and results on all estimates and the indirect effects were indeed similar.

Direction of Effects Between Maternal Criticism, Adolescent Perceived Criticism, and Adolescent Depressive Symptoms

Figure 1 graphically displays the final cross-lagged panel model for adolescent depressive symptoms. Within-wave correlations in this model ranged from 0.19 to 0.29 between maternal criticism and adolescent perceived criticism, from
0.09 to 0.23 between maternal criticism and depressive symptoms, and from 0.27 to 0.42 between adolescent perceived criticism and depressive symptoms. In this model, we found significant bidirectional effects (i.e., both parent and child effects) between maternal criticism, adolescent perceived criticism, and adolescent depressive symptoms. That is, maternal criticism predicted higher levels of adolescent perceived criticism in the subsequent year, and adolescent perceived criticism predicted higher levels of depressive symptoms in the subsequent year, thereby providing support for a parent effects model. Furthermore, adolescent depressive symptoms predicted higher levels of adolescent perceived criticism in the subsequent year, and adolescent perceived criticism predicted higher levels of maternal criticism in the subsequent year, thereby providing support for a child effects model. In sum, we found support for a reciprocal effects model, including both parent effects and child effects in the association between maternal criticism and adolescent depressive symptoms, via adolescents' perceptions of criticism.

Significant longitudinal mediation was also observed from maternal criticism to adolescent depressive symptoms, via adolescent perceived criticism, $b=0.004, S E=0.002, \beta=$ $0.003, p=0.038$, as well as the reverse from adolescent depressive symptoms to maternal criticism, via adolescent perceived criticism, $b=0.010, S E=0.002, \beta=0.013 / 0.014$, $p<0.001$. As hypothesized, we found the child effects mediation from adolescent depressive symptoms to maternal criticism, via adolescent perceived criticism, to be significantly stronger, $\Delta b=0.006, \Delta \beta=0.010,95 \% \mathrm{CI}=0.001$, 0.011 (one-tailed), than the parent effects mediation from maternal criticism to adolescent depressive symptoms, via adolescent perceived criticism (using the analytical strategy for comparing differences between indirect effects described in Preacher and Hayes 2008).

Direction of Effects Between Maternal Criticism, Adolescent Perceived Criticism, and Adolescent Generalized Anxiety Disorder Symptoms

Figure 2 graphically displays the final cross-lagged panel model for adolescent GAD symptoms. Within-wave correlations in this model ranged from 0.19 to 0.29 between maternal criticism and adolescent perceived criticism, from 0.01 to 0.15 between maternal criticism and GAD symptoms, and from 0.13 to 0.26 between adolescent perceived criticism and GAD symptoms. In this model, we found significant child effects, but no significant parent effects between maternal criticism and adolescent GAD symptoms. That is, maternal criticism predicted higher levels of adolescent perceived criticism in the subsequent year, but adolescent perceived criticism did not predict levels of GAD symptoms in the subsequent year. Furthermore, adolescent GAD symptoms predicted higher levels of adolescent 
Fig. 1 Standardized associations between maternal criticism, adolescent perceived criticism, and adolescent depressive symptoms over time. Although not displayed for reasons of clarity, this model includes associations between all variables within waves $\left(T_{1}\right.$ correlations and correlated change) and 2 year stability paths

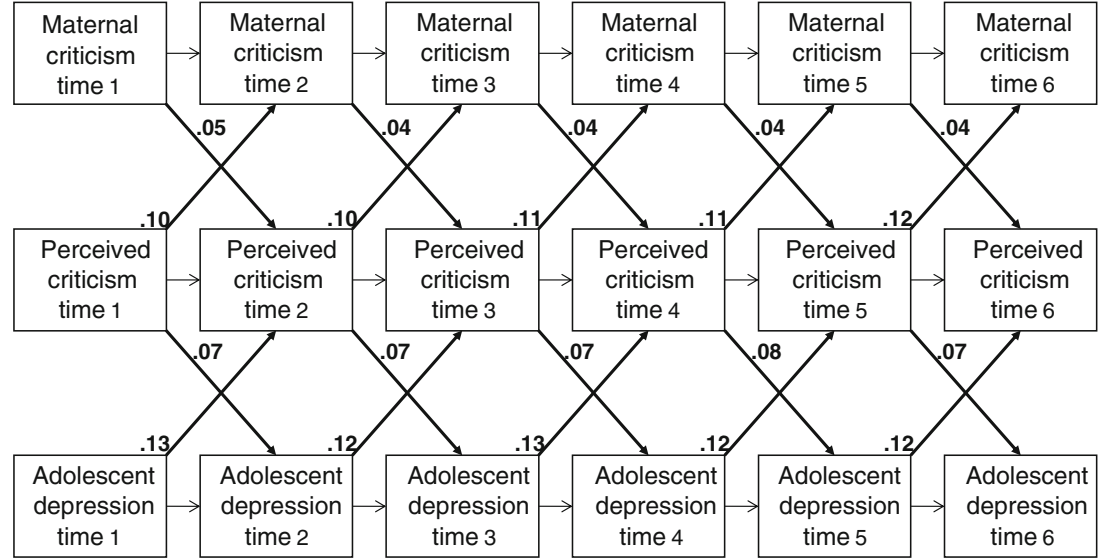

perceived criticism in the subsequent year, and adolescent perceived criticism predicted higher levels of maternal criticism in the subsequent year, thereby providing support for a child effects model. Significant longitudinal mediation was observed from adolescent GAD symptoms to maternal criticism, via adolescent perceived criticism, $b=0.010, S E=0.003, \beta=$ $0.010 / 0.011, p<0.001$. In sum, we found support for a child effects model in the association between maternal criticism and adolescent GAD symptoms, via adolescent perceived criticism.

\section{Discussion}

This 6-year longitudinal community study prospectively examined the direction of effects (i.e., parent effects, child effects, or reciprocal effects) between maternal criticism and adolescent depressive and GAD symptoms, including adolescent perceived criticism as a potential mediator. Our results provide preliminary support for a reciprocal effects model (i.e., both parent effects and child effects) with respect to maternal criticism and adolescent depressive symptoms, mediated by adolescent perceived criticism (Fig. 1). As expected, we found stronger child effects mediation than parent effects mediation. That is, adolescent depressive symptoms were a stronger predictor of later maternal criticism, via adolescent perceived criticism, than the reverse. Furthermore, our results provide preliminary support for a child effects model only with respect to maternal criticism and adolescent GAD symptoms, via adolescent perceived criticism (Fig. 2). In other words, adolescent GAD symptoms were predictive of later maternal criticism and this association was mediated by adolescent perceived criticism. Overall, our results suggest stronger support for child effects than parent effects in the longitudinal associations between maternal criticism, adolescent perceived criticism, and adolescent symptoms of depression and GAD from early to late adolescence.

\section{Direction of Effects: Parent Effects, Child Effects, or Reciprocal Effects}

While theoretical models have long emphasized the importance of considering bidirectional parent-child effects (Bell 1968) and comparable theoretical suggestions have been made with regard to parental EE/criticism and adolescent psychopathological symptoms specifically (e.g., Asarnow et al. 2001; McCarty et al. 2004), this is one of the first
Fig. 2 Standardized associations between maternal criticism, adolescent perceived criticism, and adolescent Generalized Anxiety Disorder (GAD) symptoms over time. Although not displayed for reasons of clarity, this model includes associations between all variables within waves $\left(T_{1}\right.$ correlations and correlated change) and 2 year stability paths

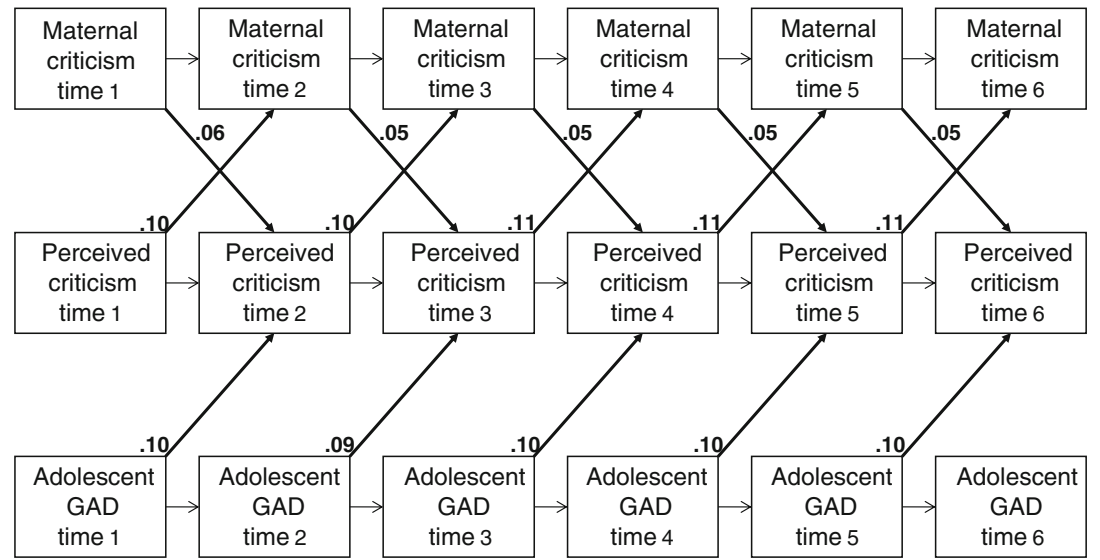


empirical studies to longitudinally examine the direction of effects between a questionnaire measure of maternal criticism and adolescent symptoms of anxiety and depression in a community sample. Even though parent effects have been most commonly studied, child effects or reciprocal effects may be especially important to study during the realignment of the parent-child relationship in adolescence. As the parentadolescent relationship becomes more egalitarian and reciprocal (De Goede et al. 2009), parental criticism may be less likely to affect adolescent characteristics (i.e., parent effects) than adolescent symptoms of anxiety or depression may be to elicit or intensify particular patterns of parental responding (i.e., child effects).

In our study, we found stronger support for child effects than parent effects in the longitudinal associations between maternal criticism and both adolescent depression and GAD. This suggests that previous studies have provided us with an incomplete picture regarding this association by solely focusing on parent effects. In addition, our results further emphasize the importance of examining potential child effects in addition to parent effects in associations between aspects of the parentchild relationship and adolescent psychopathological symptoms. However, we should note that the reliability of maternal criticism in the present study was found to be consistently unsatisfactory (Cronbach's $\alpha=0.57-0.59$ over time). Since the psychometric properties of the LEE questionnaire have been verified in previous studies (Gerlsma and Hale 1997; Hale et al. 2007), this may reflect reporter bias specific to the maternal reports of the mother-adolescent relationship in our sample, although the measure did demonstrate high rank-order stability and satisfactory concurrent validity (e.g., moderate correlations with adolescent perceived criticism and depressive symptoms) over time. Generally, low reliability coefficients make it more difficult to find statistically significant paths, so our results may be an underestimation of the associations between maternal reports of criticism and adolescent perceived criticism and psychopathological symptoms.

Our results fit well with recent findings on associations between other aspects of parenting and adolescent anxiety and depressive symptoms that have found stronger child effects than parent effects when considering potential bidirectional associations (e.g., Branje et al. 2010; Van Eijck et al. 2012; Wijsbroek et al. 2011). With respect to adolescent depressive symptoms, our results seem to suggest an ongoing downward spiral. Higher levels of a negative emotional climate between parents and adolescents appear to indirectly predict higher levels of later adolescent depressive symptoms, while at the same time adolescent depressive symptoms indirectly reinforce a later negative emotional climate between parents and their adolescents. The results seem to suggest a slightly different pattern with respect to adolescent GAD symptoms, where adolescent symptoms mainly appear to deteriorate the emotional climate between mothers and adolescents. Although these results may seem inconsistent with the described mean levels of maternal criticism, adolescent perceived criticism, and adolescent depressive and GAD symptoms over time, we would like to clarify that the reported deteriorations in the parent-child interaction patterns over time in our statistical models reflect rank-order changes and not mean level changes over time.

Our stronger support for child effects, compared to parent effects, for both adolescent depression and GAD further fits within Coyne's (1976) interactional theory of depression, and these results could be taken as further support for an explanatory process of relational erosion that would apply to both adolescent anxiety and depression (Branje et al. 2010; Joiner and Coyne 1999). The relationship erosion perspective suggests that while high symptoms of anxiety and depression in children may initially elicit supportive parental behaviors, these children are assumed to rely on negative interpersonal interaction styles (including excessive reassurance seeking) that induce a negative mood in their interaction partners. Hence, through a process of support erosion, the initially supportive parent-adolescent interaction becomes increasingly rejecting and negative, including higher levels of EE/criticism. This suggests not only that higher levels of maternal criticism and higher levels of adolescent anxiety and depression co-occur (i.e., a co-occurrence of problems), but also that higher levels of adolescent depressive and GAD symptoms appear to erode the parent-child relationship over time.

These results could have potential clinical implications, but we should be cautious in discussing these because this study was conducted in a community sample. Even though the term "child effects" may seem to imply that adolescents themselves should be the focus of intervention practices, adolescents' anxiety and depressive symptoms appear to have an important negative effect on the parent-adolescent relationship. Therefore, including parents in interventions for adolescent problems of anxiety and depression may be recommended to ameliorate both negative family dynamics and adolescent anxiety and depressive symptoms. This has been suggested previously by other researchers (e.g., discussed in Bögels and Siqueland 2006; Restifo and Bögels 2009). Future research is needed, however, to replicate these findings and further explore associations between parental behaviors and attitudes and adolescent psychopathology.

\section{Mediating Role of Adolescent Perceived Criticism}

In line with previous reasoning (Millikan et al. 2002; Powers et al. 1994; Rohner et al. 2005; Yahav 2007), our results suggest that adolescents' perceptions of parental criticism are a critical mediating variable in associations between maternal criticism and adolescent symptoms of depression and GAD. In fact, we found no direct longitudinal associations between maternal 
criticism and adolescent symptoms including adolescent perceived criticism in our models. For adolescent depressive symptoms, this suggests that adolescents' perceptions of the parenting they receive are important to consider, as adolescents' subjective experiences of criticism appeared to have a stronger influence on their symptoms of depression than the criticism they received according to their mothers. As formulated by Gecas and Schwalbe (1986, p. 42): "It is our perception of others' attitude or behavior which are more consequential for our own attitudes and behavior than the actual attitudes or behavior of others". Other researchers have suggested that adolescents may internalize (perceived) parental criticism as negative self-evaluation or self-criticism, which in turn is associated with increased depressive feelings (Bolton et al. 2009; Brewin et al. 1992, 1996).

However, since we found stronger support for child effects than for parent effects in associations between maternal criticism and both adolescent depressive and GAD symptoms, this also points towards a critical role of adolescents' subjective experiences of criticism in mothers' views of their own criticism. These child effects are in line with suggestions that adolescent psychopathological symptoms may negatively affect perceptions of the self (Bolton et al. 2009) and the parent-adolescent relationship, which in turn may (further) deteriorate parents' perceptions of the parent-adolescent emotional climate (e.g., Bögels and Brechman-Toussaint 2006; Yahav 2007). It has been suggested that negative self-evaluations could predispose adolescents to process social experiences in a manner consistent with their self-worth, so that parental experiences are more likely to be interpreted as critical (Bolton et al. 2009). Adolescents may act upon these subjective negative appreciations of the parentadolescent emotional climate, thereby reinforcing the existing critical emotional climate or negative parent-child interaction pattern. Mothers, in turn, likely react to this with increased criticism (i.e., relational erosion). On the other hand, adolescents' negative appreciations of the parent-adolescent emotional climate may mirror objectively more difficult interactions with their parents because of their internalizing symptomatology (Bögels and Brechman-Toussaint 2006), thus explaining why these perceptions predict later mother-reported criticism (i.e., accurate adolescent perception of deteriorating parent-child interaction).

In addition, we may have to consider this longitudinal prediction of maternal self-reported criticism by adolescent perceived criticism less from a parent or child effects model, and instead more with respect to entire estimated models (i.e., top part of Figs. 1 and 2), as there appeared to be an interesting dynamic interplay between maternal criticism and adolescents' perceived criticism over time. This continuous reinforcement of the negative mother-adolescent emotional climate or interaction pattern over time, fueled by adolescent symptoms of depression or GAD, may indicate that a process of "spillover" occurs between mothers' and adolescents' (negative) appreciations of the parent-adolescent emotional climate. However, we do not know of any previous studies that have examined potential longitudinal bidirectional associations between adolescent perceptions and parental perceptions of parenting or the parent-child relationship, so future research is needed to further examine this association. Most importantly, our results on the mediating role of adolescent perceived criticism suggest that researchers should recognize the importance of adolescents' subjective perceptions of parenting. Although many efforts are being made to measure parenting practices as objectively as possible, adolescents' subjective perceptions of parenting are clearly also important to consider when examining associations between aspects of the parentadolescent relationship and adolescent mental health.

\section{Strengths and Limitations}

An important strength of our study was its 6-year longitudinal design, which allowed us to disentangle the direction of effects between maternal criticism and adolescent depressive and GAD symptoms from early to late adolescence. Another important strength of our study was the multi-informant assessment of parental criticism, including independent reports of parental criticism by both mothers and adolescents. Considering both mothers' and adolescents' perceptions of parental criticism allowed us to examine the dynamic interplay between maternal self-reported criticism and adolescents' perceived parental criticism over time in association with adolescent depressive and GAD symptoms. More specifically, we were able to examine the potential mediating role of adolescent perceived criticism in associations between maternal criticism and adolescent depressive and GAD symptoms.

Our results should, however, be considered in light of some limitations. First, the cross-lagged path models used in our study only allow for inferences about temporal associations, and we cannot draw any causal conclusions from these models. Second, all of the reported cross-lagged effects were small in terms of effect size. It is, however, important to consider that the reported models are complex in nature and that from this point of view it is fairly impressive to find even small 1 year longitudinal cross-lagged effects after including 1 year and 2 year stability paths and six waves of within-wave correlations, thereby controlling for these associations. Third, our results are limited by a focus on maternal criticism only, and did not include paternal criticism. Future research should involve fathers, since previous research has shown that mothers and fathers uniquely contribute to the development and maintenance of children's anxiety (Bögels and Phares 2008).

Furthermore, our focus on adolescents from the general population limits the generalizability of our results to clinical populations. In addition, caution is necessary with the generalizability of our results to other (especially nonWestern) populations since our sample only included ethnic Dutch adolescents. Even though our results could potentially have cross-cultural validity in other Western societies, the 
meaning of EE is likely to be influenced by a broad array of cultural factors that likely limit the generalizability of our results to non-Western societies (Bhugra and McKenzie 2003; Jenkins and Karno 1992). Finally, our sole reliance on questionnaires as method of assessment could be considered a limitation of the present study. Although the traditional assessment method of EE has been the Camberwell Family Interview (CFI; Vaughn and Leff 1976), and to a lesser extent the Five Minute Speech Sample (FMSS; Magana et al. 1986) interview, Kavanagh (1992) has argued the EE concept should not rest on a single operationalization. The LEE questionnaire has been suggested as a good alternative assessment method in several reviews (e.g., Kazarian 1992; Van Humbeeck et al. 2002), though we acknowledge that interviews could provide important additional information regarding EE criticism.

\section{Conclusion}

Concluding, in this 6-year longitudinal community study we found preliminary support for a reciprocal effects model (i.e., both parent effects and child effects) with respect to maternal criticism and adolescent depressive symptoms, and a child effects model with respect to maternal criticism and adolescent GAD symptoms. Furthermore, we found adolescent perceived criticism to act as a significant longitudinal mediator in these associations. Thus we found stronger support for child effects than parent effects in the longitudinal associations between maternal criticism and adolescent internalizing symptoms, including adolescents' perception of parental criticism as a critical mediating variable. This study thereby further emphasizes the importance of examining potential child effects in addition to parent effects in associations between aspects of the parent-child relationship and adolescent psychopathological symptoms, and suggests that higher levels of maternal criticism and higher levels of adolescent anxiety and depression do not only co-occur (i.e., a co-occurrence of problems), but also that higher levels of adolescent depressive and GAD symptoms appear to erode the parent-child relationship over time.

Acknowledgment Data of the RADAR study were used. RADAR has been financially supported by main grants from the Netherlands Organisation for Scientific Research (GB-MAGW 480-03-005, GBMAGW 480-08-006), and Stichting Achmea Slachtoffer en Samenleving (SASS), and various other grants from the Netherlands Organisation for Scientific Research, the VU University Amsterdam and Utrecht University.

\section{References}

American Psychiatric Association. (2000). Diagnostic and statistical manual of mental disorders, 4th ed., Text Revision (DSM-IV-TR). Washington, DC: American Psychiatric Association.
Asarnow, J. R., Goldstein, M. J., Tompson, M., \& Guthrie, D. (1993). One-year outcomes of depressive disorders in child psychiatric inpatients: evaluation of the prognostic power of a brief measure of expressed emotion. Journal of Child Psychology and Psychiatry, 34, 129-137. doi:10.1111/j.1469-7610.1993.tb00975.x.

Asarnow, J. R., Tompson, M., Woo, S., \& Cantwell, D. P. (2001). Is expressed emotion a specific risk factor for depression or a nonspecific correlate of psychopathology? Journal of Abnormal Child Psychology, 29, 573-583. doi:10.1023/A:1012237411007.

Bell, R. Q. (1968). A reinterpretation of the direction of effects in studies of socialization. Psychological Review, 75, 81-95. doi:10.1037/ h0025583.

Bhugra, D., \& McKenzie, K. (2003). Expressed emotion across cultures. Advances in Psychiatric Treatment, 9, 342-348. doi:10.1192/apt.9.5.342.

Birmaher, B., Khetarpal, S., Brent, D., Cully, M., Balach, L., Kaufman, J., et al. (1997). The Screen for Child Anxiety Related Emotional Disorders (SCARED): scale construction and psychometric characteristics. Journal of the American Academy of Child and Adolescent Psychiatry, 36, 545-553. doi:10.1097/00004583199704000-00018.

Bögels, S. M., \& Brechman-Toussaint, M. L. (2006). Family issues in child anxiety: attachment, family functioning, parental rearing and beliefs. Clinical Psychology Review, 26, 834-856. doi:10.1016/ j.cpr.2005.08.001.

Bögels, S. M., \& Phares, V. (2008). Fathers' role in the etiology, prevention and treatment of child anxiety: a review and new model. Clinical Psychology Review, 28, 539-558. doi:10.1016/j.cpr.2007. 07.011.

Bögels, S. M., \& Siqueland, L. (2006). Family cognitive behavioral therapy for children and adolescents with clinical anxiety disorders. Journal of the American Academy of Child and Adolescent Psychiatry, 45, 134 141. doi:10.1097/01.chi.0000190467.01072.ee.

Bolton, C., Barrowclough, C., \& Calam, R. (2009). Parental criticism and adolescent depression: does adolescent self-evaluation act as a mediator? Behavioural and Cognitive Psychotherapy, 37, 553570. doi:10.1017/S1352465809990221.

Brady, E. U., \& Kendall, P. C. (1992). Comorbidity of anxiety and depression in children and adolescents. Psychological Bulletin, 111, 244-255. doi:10.1037/0033-2909.111.2.244.

Branje, S. J. T., Hale, W. W., III, \& Meeus, W. H. J. (2008). Reciprocal development of parent-adolescent support and adolescent problem behaviors. In M. Kerr, H. Stattin, \& R. C. M. E. Engels (Eds.), What can parents do? New insights into the role of parents in adolescent problem behavior (pp. 135-162). doi:10.1002/ 9780470774113.ch6.

Branje, S. J. T., Hale, W. W., III, Frijns, T., \& Meeus, W. H. J. (2010). Longitudinal associations between perceived parent-child relationship quality and depressive symptoms in adolescence. Journal of Abnormal Child Psychology, 38, 751-763. doi:10. 1007/s10802-010-9401-6.

Brewin, C. R., Firth-Cozens, J., Furnham, A., \& McManus, C. (1992). Self-criticism in adulthood and recalled childhood experience. Journal of Abnormal Psychology, 101, 561-566. doi:10.1037/ 0021-843X.101.3.561.

Brewin, C. R., Andrews, B., \& Furnham, A. (1996). Self-critical attitudes and parental criticism in young women. British Journal of Medical Psychology, 69, 69-78. doi:10.1111/j.2044-8341.1996.tb01851.x.

Butzlaff, R. L., \& Hooley, J. M. (1998). Expressed emotion and psychiatric relapse: a meta-analysis. Archives of General Psychiatry, 55, 547-552. doi:10.1001/archpsyc.55.6.547.

Coyne, J. C. (1976). Depression and the response of others. Journal of Abnormal Psychology, 85, 186-193. doi:10.1037//0021-843X.85.2.186.

De Goede, I. H. A., Branje, S. J. T., \& Meeus, W. H. J. (2009). Developmental changes in adolescents' perceptions of relationships with their parents. Journal of Youth and Adolescence, 38, 75-88. doi:10.1007/s10964-008-9286-7. 
Frye, A. A., \& Garber, J. (2005). The relations among maternal depression, maternal criticism, and adolescents' externalizing and internalizing symptoms. Journal of Abnormal Child Psychology, 33, 1-11. doi:10.1007/s10802-005-0929-9.

Gar, N. S., \& Hudson, J. L. (2008). An examination of the interactions between mothers and children with anxiety disorders. Behaviour Research and Therapy, 46, 1266-1274. doi:10.1016/j.brat.2008.08. 006.

Gar, N. S., \& Hudson, J. L. (2009). Changes in maternal expressed emotion toward clinically anxious children following cognitive behavioral therapy. Journal of Experimental Child Psychology, 104, 346-352. doi:10.1016/j.jecp.2009.06.001.

Gecas, V., \& Schwalbe, M. L. (1986). Parental behavior and adolescent self-esteem. Journal of Marriage and Family, 48, 37-46. doi:10. 2307/352226.

Gerlsma, C., \& Hale, W. W., III. (1997). Predictive power and construct validity of the Level of Expressed Emotion (LEE) scale. Depressed out-patients and couples from the general community. British Journal of Psychiatry, 170, 520-525. doi:10.1192/bjp.170.6.520.

Hale, W. W., III, Raaijmakers, Q. A. W., Muris, P., \& Meeus, W. H. J. (2005). Psychometric properties of the Screen for Child Anxiety Related Emotional Disorders (SCARED) in the general adolescent population. Journal of the American Academy of Child and Adolescent Psychiatry, 44, 283-290. doi:10.1097/00004583200503000-00013.

Hale, W. W., III, Raaijmakers, Q., Gerlsma, C., \& Meeus, W. (2007). Psychometric properties of the Level of Expressed Emotion (LEE) questionnaire for adolescents from the general population. Journal of Social Psychiatry and Psychiatric Epidemiology, 42, 215-220. doi:10.1007/s00127-006-0145-0.

Hale, W. W., III, Raaijmakers, Q. A. W., Muris, P., Van Hoof, A., \& Meeus, W. H. J. (2009). One factor or two parallel processes? Comorbidity and development of adolescent anxiety and depressive disorder symptoms. Journal of Child Psychology and Psychiatry, 50, 1218-1226. doi:10.1111/j.1469-7610.2009.02115.x.

Hale, W. W., III, Crocetti, E., Raaijmakers, Q. A. W., \& Meeus, W. H. J. (2011a). A meta-analysis of the cross-cultural psychometric properties of the Screen for Child Anxiety Related Emotional Disorders (SCARED). Journal of Child Psychology and Psychiatry, 52, 80-90. doi:10.1111/j.1469-7610.2010.02285.x.

Hale, W. W., III, Keijsers, L., Klimstra, T. A., Raaijmakers, Q. A. W., Hawk, S., Branje, S. J. T., et al. (2011b). How does longitudinally measured maternal expressed emotion affect internalizing and externalizing symptoms of adolescents from the general community? Journal of Child Psychology and Psychiatry, 52, 1174-1183. doi:10.1111/j.1469-7610.2011.02400.x.

Hale, W. W., III, Raaijmakers, Q. A. W., Van Hoof, A., \& Meeus, W. H. J. (2011c). The predictive capacity of perceived expressed emotion as a dynamic entity of adolescents from the general community. Social Psychiatry and Psychiatric Epidemiology, 46, 507-515. doi:10. 1007/s00127-010-0218-y.

Holden, G. W., \& Miller, P. C. (1999). Enduring and different: a metaanalysis of the similarity in parents' child rearing. Psychological Bulletin, 125, 223-254. doi:10.1037//0033-2909.125.2.223.

Hooley, J. M., \& Teasdale, J. D. (1989). Predictors of relapse in unipolar depressives: expressed emotion, marital distress, and perceived criticism. Journal of Abnormal Psychology, 98, 229-235. doi:10. 1037//0021-843X.98.3.229.

Hughes, E. K., \& Gullone, E. (2008). Internalizing symptoms and disorders in families of adolescents: a review of family systems literature. Clinical Psychology Review, 28, 92-117. doi:10.1016/j. cpr.2007.04.002.

Jenkins, J. H., \& Karno, M. (1992). The meaning of expressed emotion: theoretical issues raised by cross- cultural research. American Journal of Psychiatry, 149, 9-21. Retrieved from http://ajp. psychiatryonline.org.
Joiner, T. E., \& Coyne, J. C. (1999). The interactional nature of depression: Advances in interpersonal approaches. Washington: American Psychiatric Association.

Kavanagh, D. J. (1992). Recent developments in expressed emotion and schizophrenia. British Journal of Psychiatry, 160, 601-620. doi:10. 1192/bjp.160.5.601.

Kazarian, S. S. (1992). The measurement of expressed emotion: a review. Canadian Journal of Psychiatry, 37(1), 51-56.

Kerr, M., \& Stattin, H. (2003). Parenting of adolescents: Action or reaction? In A. C. Crouter \& A. Booth (Eds.), Children's influence on family dynamics: The neglected side of family relationships (pp. 121-151). New York: Erlbaum. Retrieved from http://www. scribd.com.

Laursen, B., \& Collins, A. W. (2009). Parent-adolescent relationships during adolescence. In R. M. Lerner \& L. Steinberg (Eds.), Handbook of adolescent psychology (3rd ed., Vol. 2, pp. 3-42). Hoboken: Wiley.

Lollis, S., \& Kuczynski, L. (1997). Beyond one hand clapping: seeing bidirectionality in parent-child relations. Journal of Social and Personal Relationships, 14, 441-461. doi:10.1177/02654075971 44002.

Magana, A. B., Goldstein, M. J., Karno, M., Miklowitz, D. J., Jenkins, J., \& Falloon, I. R. H. (1986). A brief method for assessing expressed emotion in relatives of psychiatric patients. Psychiatry Research, 17, 203-212. doi:10.1016/0165-1781(86)90049-1.

McCarty, C. A., Lau, A. S., Valeri, S. M., \& Weisz, J. R. (2004). Parentchild interactions in relation to critical and emotionally overinvolved expressed emotion (EE): is EE a proxy for behavior? Journal of Abnormal Child Psychology, 32, 83-93. doi:10.1023/B:JACP. 0000007582.61879.6f.

McLeod, B. D., Weisz, J. R., \& Wood, J. J. (2007a). Examining the association between parenting and childhood depression: a metaanalysis. Clinical Psychology Review, 27, 986-1003. doi:10.1016/ j.cpr.2007.03.001.

McLeod, B. D., Wood, J. J., \& Weisz, J. R. (2007b). Examining the association between parenting and childhood anxiety: a metaanalysis. Clinical Psychology Review, 27, 155-172. doi:10.1016/ j.cpr.2006.09.002.

Merikangas, K. R., He, J.-P., Burstein, M., Swanson, S. A., Avenevoli, S., Cui, L., et al. (2010). Lifetime prevalence of mental disorders in U.S. adolescents: results from the national comorbidity survey replication-adolescent supplement (NCS-A). Journal of the American Academy of Child and Adolescent Psychiatry, 49, 980 989. doi:10.1016/j.jaac.2010.05.017.

Millikan, E., Wamboldt, M. Z., \& Bihun, J. T. (2002). Perceptions of the family, personality characteristics, and adolescent internalizing symptoms. Journal of the American Academy of Child and Adolescent Psychiatry, 41, 1486-1494. doi:10.1097/01.CHI. 0000024866.60748.0F.

Muthén, L. K., \& Muthén, B. O. (2010). Mplus user's guide (6th ed.). Los Angeles: Muthén \& Muthén.

Osman, A., Gutierrez, P. M., Bagge, C. L., Fang, Q., \& Emmerich, A. (2010). Reynolds adolescent depression scale-second edition: a reliable and useful instrument. Journal of Clinical Psychology, 66, 1324-1345. doi:10.1002/jclp.20727.

Powers, S. I., Welsh, D. P., \& Wright, V. (1994). Adolescents' affective experience of family behaviors: the role of subjective understanding. Journal of Research on Adolescence, 4, 585-600. doi:10.1207/ s15327795jra0404_9.

Preacher, K. J., \& Hayes, A. F. (2008). Asymptotic and resampling strategies for assessing and comparing indirect effects in multiple mediator models. Behavior Research Methods, 40, 879-891. doi: 10.3758/BRM.40.3.879.

Restifo, K., \& Bögels, S. M. (2009). Family processes in the development of youth depression: translating the evidence to treatment. Clinical Psychology Review, 29, 294-316. doi:10.1016/j.cpr.2009.02.005. 
Reynolds, W. M. (2000). Reynolds adolescent depression scale, 2nd edition (RADS-2). Professional Manual. Lutz, FL: Psychological Assessment Resources.

Rohner, R. P., Khaleque, A., \& Cournoyer, D. E. (2005). Parental acceptance-rejection: theory, methods, cross-cultural evidence, and implications. Ethos, 33, 299-334. doi:10.1525/eth.2005.33.3.299.

Schwartz, C. E., Dorer, D. J., Beardslee, W. R., Lavori, P. W., \& Keller, M. B. (1990). Maternal expressed emotion and parental affective disorder: risk for childhood depressive disorder, substance abuse, or conduct disorder. Journal of Psychiatric Research, 24, 231-250. doi:10.1016/0022-3956(90)90013-G.

Silk, J. S., Ziegler, M. L., Whalen, D. J., Dahl, R. E., Ryan, N. D., Dietz, L. J., et al. (2009). Expressed emotion in mothers of currently depressed, remitted, high-risk, and low-risk youth: links to child depression status and longitudinal course. Journal of Clinical Child and Adolescent Psychology, 38, 36-47. doi:10.1080/15374410802575339.

Tompson, M. C., Pierre, C. B., Boger, K. D., McKowen, J. W., Chan, P. T., \& Freed, R. D. (2010). Maternal depression, maternal expressed emotion, and youth psychopathology. Journal of Abnormal Child Psychology, 38, 105-117. doi:10.1007/s10802-009-9349-6.

Van Eijck, F. E. A. M., Branje, S. J. T., Hale, W. W., III, \& Meeus, W. H. J. (2012). Longitudinal associations between perceived parent- adolescent attachment relationship quality and Generalized Anxiety Disorder symptoms in adolescence. Journal of Abnormal Child Psychology, 40, 871-883. doi:10.1007/s10802-012-9613-z.

Van Humbeeck, G., Van Audenhove, C., De Hert, M., Pieters, G., \& Storms, G. (2002). Expressed emotion: a review of assessment instruments. Clinical Psychology Review, 22, 321-341. doi:10. 1016/S0272-7358(01)00098-8.

Vaughn, C., \& Leff, J. (1976). The measurement of expressed emotion in the families of psychiatric patients. British Journal of Social and Clinical Psychology, 15, 157-165. doi:10.1111/j.2044-8260.1976. tb00021.x.

Vaughn, C., \& Leff, J. (1985). Expressed emotion in families: Its significance for mental illness. New York: Guilford Press.

Wijsbroek, S. A. M., Hale, W. W., III, Raaijmakers, Q. A. W., \& Meeus, W. H. J. (2011). The direction of effects between perceived parental behavioral control and psychological control and adolescents' selfreported GAD and SAD symptoms. European Child and Adolescent Psychiatry, 20, 361-371. doi:10.1007/s00787-011-0183-3.

Yahav, R. (2007). The relationship between children's and adolescents' perceptions of parenting style and internal and external symptoms. Child: Care, Health and Development, 33, 460-471. doi:10.1111/ j.1365-2214.2006.00708. 